\title{
GENERALIZED PASCAL'S TRIANGLE AND METALLIC RATIOS
}

\section{R. Sivaraman ${ }^{1}$ ]}

${ }^{1}$ Independent Research Scholar, California Public Univeristy, USA

\section{ABSTRACT}

In this paper, I had demonstrated the way to determine the sequence of metallic ratios by generalizing the usual Pascal's triangle. In doing so, I found several interesting properties that had been discussed in detail in this paper. I had proved four new results upon generalizing Pascal's triangle. Thus, the primary aim of this paper is to connect the idea of Generalized Pascal's triangle with that to the sequence of metallic ratios.

Received 1 July 2021

Accepted 16 July 2021

Published 31 July 2021

Corresponding Author

R. Sivaraman, rsivaraman1729@y ahoo.co.in

DOI 10.29121/

granthaalayah.v9.i7.2021.4109

Funding: This research received no specific grant from any funding agency in the public, commercial, or not-for-profit sectors.

Copyright: (C) 2021 The Author(s). This is an open access article distributed under the terms of the Creative Commons Attribution License, which permits unrestricted use, distribution, and reproduction in any medium, provided the original author and source are credited.

OPEN
Keywords: Generalized Pascal's Triangle, Metallic Ratio of Order K, Binomial Coefficient, Hockey Stick Property, Binet's Formula Respresentation

\section{INTRODUCTION}

Though the concept of Pascal's triangle became notable among mathematical world through Pascal, the concept of constructing the triangle was well known to ancient Indian and Chinese mathematicians. Similarly, the sequence of metallic ratios has been used in almost all branches of Science and Engineering. In this paper, I had connected these two concepts and derived some interesting results related to them.

\section{DEFINITION}

Let $k$ be a positive integer. The sequence of Metallic ratios of order $k$ is defined recursively by $M_{n+2}=k M_{n+1}+M_{n}(2.1), n \geq 1$ where $M_{0}=0, M_{1}=1, M_{2}=k$ The terms of the sequence defined by (2.1) are given by $0,1, k, k^{2}+1, k^{3}+2 k, k^{4}+3 k^{2}+1, k^{5}+4 k^{3}+3 k, k^{6}+5 k^{4}+6 k^{2}+1, \ldots$ (2.2)

Notice that for $k=1$, the above sequence is the usual Fibonacci sequence.

\subsection{METALLIC RATIOS OF ORDER K}

Using the shift operator, the recurrence relation in (2.1), yield the quadratic equation $m^{2}-k m-1=0$ The two real roots of this quadratic equation are given by $m=$ $\frac{k \pm \sqrt{k^{2}+4}}{2}$ The positive value among these two roots is defined as the metallic ratio of 
order $k$ denoted by $\rho_{k}$ Thus, $\rho_{k}=\frac{k+\sqrt{k^{2}+4}}{2}$ (2.3) Since the sum of two roots is k, the other root is $k-\rho_{k}=\frac{k-\sqrt{k^{2}+4}}{2}(2.4)$

\subsection{SPECIAL CASES}

(i) If $k=1$, then from (2.3), $\rho_{1}=\frac{1+\sqrt{5}}{2}(2.5)$ is called the golden ratio.

(ii) If $k=2$, then from (2.3), $\rho_{2}=1+\sqrt{2}$ (2.6) is called the silver ratio.

(iii) If $k=3$, then from (2.3), $\rho_{3}=\frac{3+\sqrt{13}}{2}(2.7)$ is called the bronze ratio.

The numbers given by (2.5) to (2.7) form the metallic ratios of first three orders.

\section{CONSTRUCTION OF GENERALIZED PASCAL'S TRIANGLE}

Using the same rule of construction as that of usual Pascal's triangle, I now construct a generalized Pascal's triangle from which we can explore several properties. Let $k$ be a positive integer. Consider a triangle displayed below.

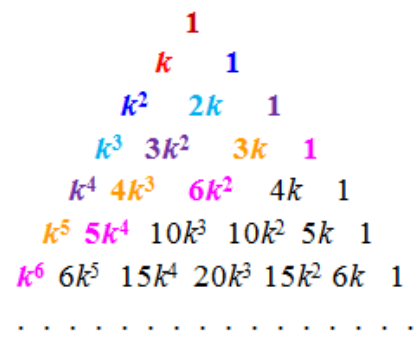

Figure 1 Generalized Pascal's Triangle

We notice that each entry in the Generalized Pascal's triangle of Figure 1 , is obtained using the recurrence relation defined in (2.1). In particular, if $T_{n, r}$ is the rth entry in $\eta$ th row, where

$n=0,1,2,3, \ldots, 0 \leq r \leq n$ and $T_{0,0}=1, T_{n, 0}=k^{n}, T_{n, n}=1$ then $T_{n, r}=k T_{n-1, r}+T_{n-1, r-1}$

If $k=1$, then (3.1) reduces to the definition of usual Pascal's triangle and Figure 1 would then become the well known Pascal's triangle. From Figure 1, we notice that the $r$ th entry in $n$th row where $0 \leq r \leq n$ of the Generalized Pascal's triangle in Figure 1 is given by $T_{n, r}=\left(\begin{array}{l}n \\ r\end{array}\right) K^{n-r}(3.2)$ where $\left(\begin{array}{l}n \\ r\end{array}\right)$ is the binomial coefficient. 


\subsection{THEOREM 1}

With respect to Generalized Pascal's triangle of Figure 1, we have the following properties

(i) The sum of all $n$th row entries is $(k+1)^{n}(3.3)$

(ii) $T_{n, r}=k^{n-2 r} T_{n, n-r}(3.4)$

(iii) (Hockey Stick Property of Generalized Pascal's Triangle): $k \sum_{r=0}^{m} T_{n+r, r}=T_{n+m+1, m}$

\section{Proof:}

The sum of all entries in nth row of Generalized Pascal's triangle is given by $\sum_{r=0}^{n} T_{n, r}=\sum_{r=}^{n}\left(\begin{array}{l}n \\ r\end{array}\right) k^{n-r}=\sum_{r=0}^{n}\left(\begin{array}{l}n \\ r\end{array}\right) k^{n-r} 1^{r}=(k+1)^{n}$. This proves (3.3)

Using (3.2), we have $K^{n-2 r} T_{n, n-r}=K^{n-2 r}\left(\begin{array}{c}n \\ n-r\end{array}\right) K^{n(n-r)}=K^{n-r}\left(\begin{array}{l}n \\ r\end{array}\right)=$ $T_{n, r}$

This proves (3.4)

Using (3.2) , we have

$$
\begin{aligned}
k \sum_{r=0}^{m} T_{n+r, r} & =k \sum_{r=0}^{m}\left(\begin{array}{c}
n+r \\
r
\end{array}\right) k^{n}=k^{n+1} \sum_{r=0}^{m}\left(\begin{array}{c}
n+r \\
r
\end{array}\right) \\
& =k^{n+1}\left[\left(\begin{array}{c}
n \\
0
\end{array}\right)+\left(\begin{array}{c}
n+1 \\
1
\end{array}\right)+\left(\begin{array}{c}
n+2 \\
2
\end{array}\right)+\cdots+\left(\begin{array}{c}
n+m-1 \\
m-1
\end{array}\right)+\left(\begin{array}{c}
n+m \\
m
\end{array}\right)\right] \\
& =k^{n+1}\left[\left(\begin{array}{c}
n+1 \\
n+1
\end{array}\right)+\left(\begin{array}{c}
n+1 \\
n
\end{array}\right)+\left(\begin{array}{c}
n+2 \\
n
\end{array}\right)+\cdots+\left(\begin{array}{c}
n+m-1 \\
n
\end{array}\right)+\left(\begin{array}{c}
n+m \\
n
\end{array}\right)\right] \\
& =k^{n+1}\left[\left(\begin{array}{c}
n+2 \\
n+1
\end{array}\right)+\left(\begin{array}{c}
n+2 \\
n
\end{array}\right)+\cdots+\left(\begin{array}{c}
n+m-1 \\
n
\end{array}\right)+\left(\begin{array}{c}
n+m \\
n
\end{array}\right)\right] \\
& =k^{n+1}\left[\left(\begin{array}{c}
n+3 \\
n+1
\end{array}\right)+\left(\begin{array}{c}
n+3 \\
n
\end{array}\right)+\cdots+\left(\begin{array}{c}
n+m-1 \\
n
\end{array}\right)+\left(\begin{array}{c}
n+m \\
n
\end{array}\right)\right] \\
& =k^{n+1}\left[\left(\begin{array}{c}
n+m-1 \\
n+1
\end{array}\right)+\left(\begin{array}{c}
n+m-1 \\
n
\end{array}\right)+\left(\begin{array}{c}
n+m \\
n
\end{array}\right)\right] \\
& =k^{n+1}\left[\left(\begin{array}{c}
n+m \\
n+1
\end{array}\right)+\left(\begin{array}{c}
n+m \\
n
\end{array}\right)\right] \\
& =k^{n+1}\left(\begin{array}{c}
n+m+1 \\
n+1
\end{array}\right)=k^{n+1}\left(\begin{array}{c}
n+m+1 \\
m
\end{array}\right)=T_{n+m+1, m}
\end{aligned}
$$

This proves (3.5) and hence completes the proof. 


\section{METALLIC RATIOS FROM GENERALIZED PASCAL'S TRIANGLE}

In this section, I demonstrate a method to obtain sequence of metallic ratios of order $\mathrm{k}$ as defined in (2.2) from the generalized Pascal's triangle described in Figure 1. First, we will rearrange the terms of the triangle in right triangle pattern as shown below.

1

k 1

$\begin{array}{lll}k^{2} & 2 k & 1\end{array}$

$k^{3} 3 k^{2} \quad 3 k \quad 1$

$k^{4} \quad 4 k^{3} \quad 6 k^{2} \quad 4 k \quad 1$

$k^{5} 5 k^{4} 10 k^{3} 10 k^{2} 5 k \quad 1$

$k^{6} 6 k^{5} 15 k^{4} 20 k^{3} 15 k^{2} 6 k \quad 1$

Figure 2 Add the identical colored terms in the triangle

If we add identical colored terms in the re-arranged triangle in North-East diagonal direction then we get

$$
1, k, k^{2}+1, k^{3}+2 k, k^{4}+3 k^{2}+1, k^{5}+4 k^{3}+3 k, k^{6}+5 k^{4}+6 k^{2}+1, \ldots
$$

We notice that the terms of above sequence precisely forms the terms of sequence of metallic ratios of order $\mathrm{k}$ as defined in (2.2). Thus, the triangle in Figure 2 , generates sequence of metallic ratio of order $k$.

\subsection{THEOREM 2}

The ratio of $(n+1)$ th term to that of $n$th term of sequence of metallic ratios of order $\mathrm{k}$ converges to the number $\rho_{k}=\frac{k+\sqrt{k^{2}+4}}{2}(4.1)$

Proof: From the recursive relation of metallic ratio of order $\mathrm{k}$ as defined in (2.1), notice that for some real numbers $\alpha$ and $\beta$ we get $M_{n}=\alpha \rho_{k}{ }^{n}+\beta\left(k-\rho_{k}\right)^{n}(4.2)$ where $\rho_{k}$ and $k-\rho_{k}$ are the real numbers given by $\rho_{k}=\frac{k+\sqrt{k^{2}+4}}{2}, k-\rho_{k}=\frac{k+\sqrt{k^{2}+4}}{2}$ Equation (4.2) is referred as Binet's formula representation for sequence of metallic ratios of order $k$

We now notice that $-1<k-\rho_{k}<1$ for all positive integers $\mathrm{k}$. Hence $\left(k-\rho_{k}\right)^{n} \rightarrow$ $0(4.3)$ as $n \rightarrow \infty$. Thus using (4.2) and (4.3), we obtain

$\lim _{n \rightarrow \infty} \frac{M_{n+1}}{M_{n}}=\lim _{n \rightarrow \infty} \frac{\alpha \rho_{k}{ }^{n+1}+\beta\left(k-\rho_{k}\right)^{n+1}}{\alpha \rho_{k}{ }^{n}+\beta\left(k-\rho_{k}\right)^{n}}=\lim _{n \rightarrow \infty} \frac{\alpha \rho_{k}{ }^{n+1}+0}{\alpha \rho_{k}{ }^{n}+0}=\rho_{k}=\frac{k+\sqrt{k^{2}+4}}{2}$

This completes the proof. 


\subsection{GOLDEN, SILVER AND BRONZE RATIOS}

As mentioned in 2.2 of section 2, we notice that the Golden, Silver and Bronze ratios are special cases of $\rho_{k} k=1,2,3$. Hence if we consider the triangle in Figure 1, with the values $k=1,2$ and 3 we can generate the terms of sequence of metallic ratios of orders 1,2 and 3 respectively whose ratio of successive terms approach to $\rho_{1}=$ $\frac{1+\sqrt{5}}{2}, \rho_{2}=1+\sqrt{2}, \rho_{3}=\frac{3+\sqrt{13}}{2}$ respectively. Thus we can produce number triangles which are generalized versions

\section{CONCLUSION}

In this paper, by defining a generalized Pascal's triangle, first, I had established three interesting properties in Theorem 1. The third property proved in (3.5) is the generalized Hockey stick property which works only if we could sum numbers in South East diagonal direction unlike in usual Pascal's in which Hockey stick property works in either South - East diagonal way or in South - West diagonal way owing to its symmetrical entries. In the generalized Pascal's triangle discussed in this paper in Figure 1, by (3.4), since the entries are not symmetrical with respect to the central vertical line, we get Hockey stick property only along South - East direction.

Further, I had obtained the terms of sequence of metallic ratios of order $k$ through the generalized Pascal's triangle in section 4. Finally, I had proved that the ratio of successive terms of sequence of metallic ratios is precisely the metallic ratio of order $k$ given by $\rho_{k}$. Thus, in this paper, I had established some new properties by generalizing the usual Pascal's triangle and in doing so, I had obtained the terms of metallic ratio sequence. By considering $k=1,2,3$ in Figure 1 , we can generate three new triangles from which produces three sequences as given in (2.2), whose ratio of successive terms approaches to Golden, Silver and Bronze ratios respectively, through the generalized Pascal's triangle, we could generate sequences whose ratio of successive terms converges to the metallic ratio of order k given by $\rho_{k}=\frac{k+\sqrt{k^{2}+4}}{2}$ The ideas discussed in this paper will add more information to the study of metallic ratios and pave way for further explorations.

\section{REFERENCES}

Gil, J. B., , \& Worley, A. (2019). Generalized Metallic Means, Fibonacci Quarterly., 57(1), 45-50. Hare, K., Prodinger, H., , \& Shallit, J. (2014). Three Series For The Generalized Golden Mean, Fibonacci Quart., 52(4), 307-313.

Krcadinac, V. (2006). A New Generalization Of The Golden Ratio. Fibonacci Quarterly., 44(4), $335-340$.

Passoja, D. (2015). Reflections On The Gold, Silver And Metallic Ratios.

Sivaraman, R. (2020). Exploring Metallic Ratios. Mathematics and Statistics, 8(4), 388-391. Retrieved from https://dx.doi.org/10.13189/ms.2020.080403 10.13189/ms.2020 .080403

Sivaraman, R. (2021a). Expressing Numbers in terms of Golden, Silver and Bronze Ratios. 
Turkish Journal of Computer and Mathematics Education (TURCOMAT), 12(2), 28762880. Retrieved from https://dx.doi.org/10.17762/turcomat.v12i2.2321 10.17762/ turcomat.v12i2.2321

Sivaraman, R. (2021b). Relation Between Terms Of Sequences And Integral Powers Of Metallic Ratios. Turkish Journal Of Physiotherapy And Rehabilitation, 32(2), 1308-1311. 